\title{
Hemoglobina glicosilada como signo de alarma
}

\begin{abstract}
Manuel Martín-Regidor, Nakaire Bonache-Castejón, María Esther Fernández-Pérez, Ana Martínez-Alonso, Gerard Sancho-Pascual Servicio de Medicina Interna. Complejo Asistencial Universitario de León. León. España
\end{abstract}

Recibido: 02/11/2018

Aceptado: 01/01/2019

En línea: 31/01/2019

Citar como: Martín-Regidor M, Bonache-Castejón N, Fernández-Pérez ME, Martínez-Alonso A, Sancho-Pascual G. Hemoglobina glicosilada como signo de alarma. Rev Esp Casos Clin Med Intern (RECCMI). 2019 (Ene); 4(Supl 1): 11-13. doi: 10.32818/reccmi.a4s1a5.

Autor para correspondencia: Manuel Martín-Regidor. manuelmartinregidor@gmail.com

\section{Palabras clave \\ $\triangleright$ Hemoglobina glicosilada \\ $\triangleright$ Síndrome general \\ $\triangleright$ Neoplasia de páncreas}

\begin{tabular}{l}
\hline Keywords \\
\hline$\triangleright$ Glycosylated hemoglobin \\
$\triangleright$ General syndrome \\
$\triangleright$ Pancreatic cancer \\
\end{tabular}

\begin{abstract}
Resumen
Mujer de 67 años diabética tipo 2, que tras varios años de buen control glucémico, con cifras de hemoglobina glicosilada (HbA1c) en torno a 6,5\%, consulta por clínica cardinal con poliuria, pérdida de peso y astenia. La analítica inicial muestra cifras de HbA1c de 14\%, razón por la que se deciden ampliar estudios. Finamente, se evidencia en colangio-resonancia magnética nuclear una masa pancreática que, tras realización de ecoendoscopia con toma de biopsias, confirma la presencia de un adenocarcinoma anaplásico de páncreas.

Abstract
A 67-year-old woman with type 2 diabetes, who after several years of good glycemic control with HbA1c around
$6.5 \%$, consulted for cardinal symptoms with polyuria, weight loss and asthenia. The initial blood test shows HbA1C
$14 \%$, which is why it is decided to extend studies. A pancreatic mass is evidently found in MR cholangiography,
which after carrying out echoendoscopy with biopsies confirms the presence of an anaplastic adenocarcinoma
of the pancreas.
\end{abstract}

\section{Puntos destacados}

$\triangleright$ Ante la aparición de cambios repentinos en el control glucémico de la diabetes, debemos plantearnos cuál es la causa desencadenante, investigar causas frecuentes, tales como la falta de adherencia, y menos comunes, como la presencia de neoplasias.

\section{Introducción}

Mujer de 67 años que consulta por síndrome constitucional.

\section{Historia clínica: antecedentes, enfermedad actual y exploración física}

- Antecedentes personales:

- No alergias medicamentosas. Trabajó como administrativa. Hipertensión arterial sin lesión de órgano diana. Diabetes mellitus (DM) tipo 2, de 15 años de evolución con adecuado control, en tratamiento con antidiabéticos no insulínicos. Dislipemia. Carcinoma ductal infiltrante de mama izquierda estadio II (pT2, N1 M0) en julio de 2012 tratado con cirugía-quimioterapia-radioterapia, con radiodermatitis, neutro- penia febril y mastitis secundaria. Portadora de reservorio de catéter. Glaucoma en seguimiento por Oftalmología. Hipotiroidismo subclínico. Artrosis severa. Artritis reumatoide en tratamiento ocasional con metotrexato.

- Intervenciones quirúrgicas: histerectomía sin ooferectomía (1997), prolapso rectal y varices.

- Tratamiento habitual: paracetamol $1 \mathrm{~g}$, trazadona $100 \mathrm{mg}$ (0-0-1), condroitín sulfato $400 \mathrm{mg}$ (2-0-0), naproxeno/esomeprazol 500/20 mg (1-0-0), simvastatina 20 mg (0-0-1), timolol 0,5 (1-0-1), candesartán/HTC 16/12,5 mg (1-0-0), metformina/sitagliptina 1.000/50 mg (1-0-1), venlafaxina 75 mg (0-0-1).

- Antecedentes familiares:

- Madre fallecida a los 73 años por ictus, DM tipo 2. Padre fallecido a los 83 años por ictus. Cinco hermanos. Una hermana fallecida por cáncer de pulmón.

- Enfermedad actual. Refiere aparición de astenia, pérdida de peso, poliuria 3-4 litros al día y polidipsia desde hace 2 meses. Además, presenta estreñimiento sin productos patológicos. Desde su última revisión hace 4 meses, no ha realizado controles glucémicos ni control de la presión arterial.

- Exploración física:

- Tensión arterial: 112/85 mmHg. Peso: 74 kg. Índice de masa corporal: 27,5. Consciente, orientada y colaboradora. Buen estado general, palidez cutaneomucosa. 
Cabeza y cuello. Normal.

- Auscultación cardíaca. Rítmica, unos 100 latidos por minuto, sin soplos. - Auscultación pulmonar. Murmullo vesicular conservado, sin ruidos sobreañadidos.

- Abdomen y extremidades. Sin alteraciones.

\section{Pruebas complementarias}

- Hemograma. Véase Tabla 1.

- Bioquímica. Véanse Tabla 2, Tabla 3 y Tabla 4.

- Orina. Cociente albúmina creatinina: $11,7 \mathrm{mg} / \mathrm{g}$.

\begin{tabular}{|c|l|}
\hline Leucocitos & $7.400 / \mathrm{mm}^{3}$ (fórmula normal) \\
\hline Hemoglobina & $10 \mathrm{~g} / \mathrm{dl}$ \\
\hline VCM & $89 \mathrm{fL}$ \\
\hline Plaquetas & $220.000 / \mathrm{mm}^{3}$ \\
\hline
\end{tabular}

Tabla 1. Hemograma

\begin{tabular}{|c|l|}
\hline Glucosa (70-110) & $138 \mathrm{mg} / \mathrm{dl}$ \\
\hline Urea (10-50) & $49 \mathrm{mg} / \mathrm{dl}$ \\
\hline Úrico $(\mathbf{2 , 4 - 5 , 7 )}$ & $4,18 \mathrm{mg} / \mathrm{dl}$ \\
\hline Cr (0,5-0,9) & $0,71 \mathrm{mg} / \mathrm{dl}$ \\
\hline FG & $>60 \mathrm{ml} / \mathrm{min}$ \\
\hline Pruebas función hepática & Normales \\
\hline Iones & Normales \\
\hline Colesterol total & $158 \mathrm{mg} / \mathrm{dl}$ \\
\hline HDL & $67 \mathrm{mg} / \mathrm{dl}$ \\
\hline LDL & $74 \mathrm{mg} / \mathrm{dl}$ \\
\hline TG & $86 \mathrm{mg} / \mathrm{dl}$ \\
\hline LDH & Normal \\
\hline HbA1c & $6,50 \%$ \\
\hline
\end{tabular}

Tabla 2. Bioquímica (20-10-2015)

\begin{tabular}{|c|l|}
\hline Glucosa & $475 \mathrm{mg} / \mathrm{dl}$ \\
\hline Urea & $42 \mathrm{mg} / \mathrm{dl}$ \\
\hline Úrico & $2,51 \mathrm{mg} / \mathrm{dl}$ \\
\hline Cr & $0,82 \mathrm{mg} / \mathrm{dl}$ \\
\hline FG & $60 \mathrm{ml} / \mathrm{min}$ \\
\hline Pruebas función hepática & Normales \\
\hline Colesterol total & $148 \mathrm{mg} / \mathrm{dl}$ \\
\hline HDL & $71 \mathrm{mg} / \mathrm{dl}$ \\
\hline LDL & $46 \mathrm{mg} / \mathrm{dl}$ \\
\hline TG & $153 \mathrm{mg} / \mathrm{dl}$ \\
\hline HbA1c (4,8-5,9) & $14 \%$ \\
\hline Ferritina (15-150) & $13 \mathrm{ng} / \mathrm{ml}$ \\
\hline IST (17-48) & $17 \%$ \\
\hline CEA (0-5) & 15,3 \\
\hline Hormonas tiroideas & Normales \\
\hline
\end{tabular}

Tabla 3. Bioquímica (11-05-2016)

\begin{tabular}{|c|l|}
\hline Glucosa & $84 \mathrm{mg} / \mathrm{dl}$ \\
\hline Urea & $66 \mathrm{mg} / \mathrm{dl}$ \\
\hline Úrico & $5,4 \mathrm{mg} / \mathrm{dl}$ \\
\hline Cr & $0,73 \mathrm{mg} / \mathrm{dl}$ \\
\hline FG & $>60 \mathrm{ml} / \mathrm{min}$ \\
\hline Pruebas función hepática & Normales \\
\hline HbA1c & $11,30 \%$ \\
\hline CEA & Normal \\
\hline CA 19,9 (0-37) & 6,7 \\
\hline
\end{tabular}

Tabla 4. Bioquímica (11/07/2016)

- Tomografía computarizada (TC) abdominal (07/07/2016). Dilatación de conducto pancreático principal hasta la zona de la cabeza, donde se identifica una posible lesión hipodensa de 1,3 cm de diámetro máximo. Dudosa lesión hipodensa en el parénquima hepático, en segmento IV adyacente a la vesícula, podría estar en relación con una zona de esteatosis focal.

- Colangio-resonancia magnética (21/07/2016). Atrofia casi completa del parénquima pancreático en el cuerpo y cola. El conducto pancreático principal está dilatado con morfología arrosariada y dilatación de ductos intrapancreáticos. El escaso tejido glandular muestra tenue realce en la fase arterial por la existencia de componente de fibrosis. En el margen posteromedial de la cabeza se observa una lesión redondeada y bien definida con la señal homogénea. Engloba al conducto pancreático principal provocando obstrucción del mismo con dilatación en el cuerpo y la cola. En el proceso uncinado y hasta la papila tiene calibre normal. En el estudio dinámico muestra comportamiento hipovascular. Mide aproximadamente $17 \mathrm{~mm}$ y contacta con la vena mesentérica superior en menos de la mitad de la circunferencia, aunque probablemente está infiltrada. No se observan alteraciones en la señal del parénquima hepático ni lesiones focales.

- Ecoendoscopia (01/08/2016). Con toma de biopsias compatibles con adenocarcinoma anaplásico de páncreas.

- Intervención (01/09/2016). Duodenopancreatectomía total con antrectomía, esplenectomía, resección de vena porta y colecistectomía.

\section{Evolución}

Se trata de una paciente con adecuado control glucémico hasta octubre de 2015, con HbA1c de 6,5\% que en revisión programada a los 4 meses presenta clínica hiperglucémica junto con glucemia basal de 475 mg/dl y HbA1c de $14 \%$.

Se inicia tratamiento con insulina y se solicitan pruebas complementarias, Ilegando al diagnóstico de adenocarcinoma anaplásico de páncreas. Es sometida a cirugía y a tratamiento con quimioterapia.

A los 7 meses, ingresa por empeoramiento del estado general, junto con dolor en flanco e hipocondrio derecho y fiebre de $38^{\circ} \mathrm{C}$. Se diagnóstica de shock séptico de origen biliar con abscesos hepáticos. Se pauta antibiótico de amplio espectro, a pesar de lo cual causa éxitus.

\section{Diagnóstico}

Adenocarcinoma anaplásico de páncreas. 


\section{Discusión y conclusiones}

La DM se relaciona con determinados tumores, entre ellos el de páncreas. El 0,8\% de los pacientes diabéticos mayores de 50 años desarrollarán cáncer de páncreas. La prevalencia de diabetes en el cáncer de páncreas varía considerablemente desde el 4\% al 65\%. El cáncer de páncreas se asocia a debut o empeoramiento diabético y suele aparecer antes del diagnóstico, como en nuestro caso. El tratamiento farmacológico para la DM 2 podría tener relación con el cáncer de páncreas². Nuestra paciente estaba con iDPP-4², si bien muchos estudios con terapias incretínicas no apoyan esta relación. No existen marcadores específicos para su diagnóstico y prevención. El deterioro rápido del control glucémico fue el signo de alarma que nos condujo al diagnóstico.

\section{Bibliografía}

1. Muniraj T, Chari ST. Diabetes and pancreatic cancer. Minerva Gastroenterol Dietol. 2012; 58(4): 331-345.

2. Andersen D, Korc M, Petersen G, Eibl G, Li D, Rickels M, et al. Diabetes, pancreatogenic diabetes, and pancreatic cancer. Diabetes. 2017; 66(5): $1103-$ 1110. 